\title{
Alpha rhythms in audition: cognitive and clinical perspectives
}

\author{
Nathan Weisz ${ }^{1,2}$, Thomas Hartmann ${ }^{1}$, Nadia Müller ${ }^{1,2}$, Isabel Lorenz ${ }^{1}$ and Jonas Obleser ${ }^{3}$ \\ ' Department of Psychology, University of Konstanz, Konstanz, Germany \\ 2 Zukunftskolleg, University of Konstanz, Konstanz, Germany \\ ${ }^{3}$ Max Planck Institute for Human Cognitive and Brain Sciences, Leipzig, Germany
}

Edited by:

Ole Jensen, Radboud University, Netherlands

\section{Reviewed by:}

Mathilde Bonnefond, University of Lyon, France

Roman Freunberger, University of

Salzburg, Austria

\section{*Correspondence:}

Nathan Weisz, Department of

Psychology, University of Konstanz,

P.O. Box D23, 78457 Konstanz,

Germany.

e-mail: nathan.weisz@uni-konstanz.de
Like the visual and the sensorimotor systems, the auditory system exhibits pronounced alphalike resting oscillatory activity. Due to the relatively small spatial extent of auditory cortical areas, this rhythmic activity is less obvious and frequently masked by non-auditory alpha-generators when recording non-invasively using magnetoencephalography (MEG) or electroencephalography (EEG). Following stimulation with sounds, marked desynchronizations can be observed between 6 and $12 \mathrm{~Hz}$, which can be localized to the auditory cortex. However knowledge about the functional relevance of the auditory alpha rhythm has remained scarce so far. Results from the visual and sensorimotor system have fuelled the hypothesis of alpha activity reflecting a state of functional inhibition. The current article pursues several intentions: (1) Firstly we review and present own evidence (MEG, EEG, sEEG) for the existence of an auditory alpha-like rhythm independent of visual or motor generators, something that is occasionally met with skepticism. (2) In a second part we will discuss tinnitus and how this audiological symptom may relate to reduced background alpha. The clinical part will give an introduction into a method which aims to modulate neurophysiological activity hypothesized to underlie this distressing disorder. Using neurofeedback, one is able to directly target relevant oscillatory activity. Preliminary data point to a high potential of this approach for treating tinnitus. (3) Finally, in a cognitive neuroscientific part we will show that auditory alpha is modulated by anticipation/expectations with and without auditory stimulation. We will also introduce ideas and initial evidence that alpha oscillations are involved in the most complex capability of the auditory system, namely speech perception. The evidence presented in this article corroborates findings from other modalities, indicating that alpha-like activity functionally has an universal inhibitory role across sensory modalities.

Keywords: auditory, alpha, tau, attention, speech, tinnitus, MEG, EEG

\section{INTRODUCTION}

The most basic to the most sophisticated cognitive function is the outcome of the complex interplay within as well as between large - mostly cortical - neuronal assemblies. Interestingly by sheer numbers, the cortex is a system that is tipped toward excitation that, if not balanced by an effective inhibitory system, would create states of hyperexcitation/-synchrony out of which no useful brain function could emerge (Buzsaki et al., 2007; van Dijk et al., 2009). Normal cognitive functioning thus requires a delicate balance between excitatory and inhibitory influences, which can be flexibly adapted in order for the system to cope with situational demands. In recent years, an accumulating amount of evidence suggests that on a sensory and motor level, the excitatory-inhibitory (E-I) balance is reflected by alpha-like oscillations $(\sim 8-12 \mathrm{~Hz})$ which can be recorded non-invasively using techniques such as magnetoencephalography (MEG) or electroencephalography (EEG). In sensory regions, alpha-like oscillations are the dominant rhythm at rest and decreases in power (desynchronizes) when actively engaged in processing or even anticipating a stimulus (Thut et al., 2006). Interestingly works on visual/somatosensory attention (Foxe et al., 1998; Worden et al., 2000; Fu et al., 2001; Rihs et al., 2007) and working memory have demonstrated increases of alpha in brain regions that need to be disengaged (e.g., processing a distractor; Jokisch and Jensen, 2007; van Dijk et al., 2008; Haegens et al., 2010), indicating that the view of alpha as "idling rhythm" irrelevant to cognitive functioning is flawed. Instead, the level of alpha activity may be viewed as an indicator at which state the respective cortical tissue is along a continuum marked by excitation and inhibition: low levels of alpha reflect a state of excitation, whereas high levels of alpha a state of inhibition (Klimesch et al., 2007; Weisz et al., 2007a). While this concept is comparably well established in the visual and somatosensory domain, only very little is known about the auditory alpha rhythm, which is sometimes referred to as "tau rhythm" (auditory alpha; Lehtelä et al., 1997). This article aims to give an overview of some of our recent work in this domain. Note that we refrain from sublabeling the alpha-like rhythms according to different modalities or cognitive domains, reflecting our tenet that these rhythms are functionally similar across modalities.

The goal of the first part of the manuscript is to establish the existence of an auditory alpha rhythm. With regards to its functional relevance, our initial motivation to view auditory alpha as a rhythm reflecting the E-I balance in auditory cortex came from clinical works on tinnitus; these will constitute the second part of this article. There we will also provide tentative evidence that a modulation of ongoing alpha activity via neurofeedback techniques can be beneficial in diminishing tinnitus symptoms. Tinnitus constitutes a chronic disturbance of the E-I balance which can be viewed as pathological. However, as pointed out above, the 
E-I balance needs to be transiently flexible enough in order to meet with situational demands. Therefore in the third part of the manuscript we will speculate which functional role auditory alpha could play for auditory cognitive functions backed up by preliminary data from our labs in two important domains: The first concerns spatial attention and the influence of anticipation on perception, which are not unique to the auditory systems and which may help to delineate functional similarities of alpha rhythms across sensory modalities. The second and perhaps most outstanding feature of the auditory system is the ability to comprehend spoken language, which involves mechanisms of successfully processing the acoustic signal - often under adverse conditions such as degraded speech or irrelevant acoustic input. We will conclude this article by stressing the similarities of the auditory alpha rhythm to those recorded in visual and somatosensory regions and will delineate the most challenging future issues.

\section{ONGOING AUDITORY ALPHA ACTIVITY}

An important prerequisite for this review is the certainty that something like an "auditory alpha" indeed exists. Contrary to visual, but also somatosensory alpha (e.g., when looking at planar gradients in MEG or Laplacians in EEG), auditory alpha activity is not obviously observable from the raw data when looking at surface topographies. Unfortunately, published reports that convincingly show alpha band modulations in the auditory modality have been rare. This sometimes has lead to some skepticism whether an auditory alpha exists at all that is distinct from the visual or sensori- and motor system. We do not adhere to this pessimistic view and will summarize some evidence for the existence of such a resting rhythm.

As stated above, "resting" sensory regions, i.e., regions not subject to any adequate sensory stimulation, are marked by strong alphalike oscillations. The visual alpha activity is so strong that it can be observed in most cases by simply looking at the raw data and their associated topographies. This is partly accountable by the sheer size of the visual system which is approximately an order larger than the auditory system (volume estimates for primary visual cortex $\sim 5300 \mathrm{~mm}^{3}$ and primary auditory cortex $\sim 850 \mathrm{~mm}^{3}$; not considering hemispheric asymmetries; see Andrews et al., 1997; ArtachoPerula et al., 2004 respectively). The small size of auditory cortex makes it plausible that a potential auditory alpha rhythm leaves weaker traces on the scalp surface and is furthermore blurred by the simultaneous visual and sensori- and motor alpha rhythms. Furthermore, contrary to the visual domain where one can "close one's eyes" to visual stimulation one cannot "close one's ears" to acoustic stimulation without using the hands or external devices. In evolutionary terms this means that the auditory system is the more "attentive" modality, constantly monitoring the environment and guiding our attention to potentially salient events (orienting reaction). Since attention and modulations of alpha activity are intimately related as pointed out later, this feature of the auditory system may also account for smaller resting alpha rhythms in the auditory cortex. Nevertheless, if a similar alpha rhythm also exists in the auditory as in the visual and sensori- and motor modality then a stimulation with sound should lead to a desynchronization, i.e., power decrease of ongoing alpha activity in auditory brain regions. Since desynchronizations are always relative to some (usually pre-stimulus) baseline, they are a direct evidence that a baseline alpha rhythm exists. A seminal report on this issue was published by Lehtelä et al. (1997; see also Tiihonen et al., 1991) investigating temporal spectral evolution (TSE) of neuromagnetically recorded alpha activity following $500 \mathrm{~ms}$ bursts of white noise (see left panel of Figure 1A). Using planar gradients, which yield the strongest signal just above the actually active cortical regions, the authors found a transient decrease of alpha-like activity in the 6.5-9 Hz band following sound onset in eight out of nine participants. Furthermore in two participants showing the strongest modulations they were able to localize the desynchronization to superior temporal regions suggesting an auditory cortical generator of the baseline alpha rhythm (see right panel of Figure 1B). Importantly, neither the topographies of the planar gradient fields nor the source analysis results pointed to reactivity of visual/sensori- and motor alphagenerators, arguing for a distinct source of auditory cortical alpha.

Unfortunately, following these initial positive findings, auditory alpha activity has no longer been a strong focus of neuroscientific research. In this section, we therefore want to add some evidence from our works confirming the basic existence of an auditory alpha rhythm: (a) The most direct manner to test for the existence of an auditory alpha rhythm is to have electrodes placed in respective auditory cortical regions. In humans this is possible only in very rare cases and clinical indications, for example in patients with medication resistant epilepsy implanted with electrodes for presurgical diagnostics. In such patients with electrodes implanted directly in auditory cortical regions for stereotactical EEG, almost all patients exhibit low-frequency theta to alpha band (up to $\sim 10 \mathrm{~Hz}$ ) desynchronizations starting approximately $300 \mathrm{~ms}$ following the onset of an auditory stimulus (O. Bertrand, personal communication). One example of a patient stimulated with a 50-ms sound and an electrode in primary auditory cortex is shown in Figure 1B. Data was obtained from a classical auditory oddball paradigm, in which participants had to detect rare frequency deviants (10\%). The timefrequency representation in Figure 1B shows the oscillatory dynamics induced by the standard sound. Note that the brief stimulus is not ideal, since both the evoked onset and offset response overlap with the period of desynchronization. Nevertheless the intracranial and especially depth recordings leave no doubt that an auditory alpha-like rhythm exists, however it is a different question whether it and modulations thereof can be picked up extracranially. The study cited above (Lehtelä et al., 1997) is strong evidence in this direction. Furthermore, based on multiple studies employing auditory stimulation in our labs we are convinced that it is feasible to uncover auditory alpha using non-invasive tools. (b) For example in a recent study investigating the impact of repetitive TMS (rTMS) on sound related brain activity, we recorded EEG activity from 30 participants. In Figure 1C we display the grand average of a "virtual sensor" in right auditory cortex toward an $800 \mathrm{~ms} 40 \mathrm{~Hz}$ amplitude modulated sound. Again it becomes evident that low frequencies decrease in power in an alpha-like frequency band $(\sim 7-12 \mathrm{~Hz})$. (c) Another non-invasive tool suitable to monitor auditory cortical alpha band dynamics is $M E G$, for which an example is displayed in Figure 1D. In this example from a spatial attention experiment, desynchronization over left temporal sensors $(\sim 10-12 \mathrm{~Hz})$ is shown following an auditory cue to the right ear. The task of the participant was to report the ear on which a $42-\mathrm{Hz}$ modulated sound was presented, while a $19-\mathrm{Hz}$ distracter was presented on the 


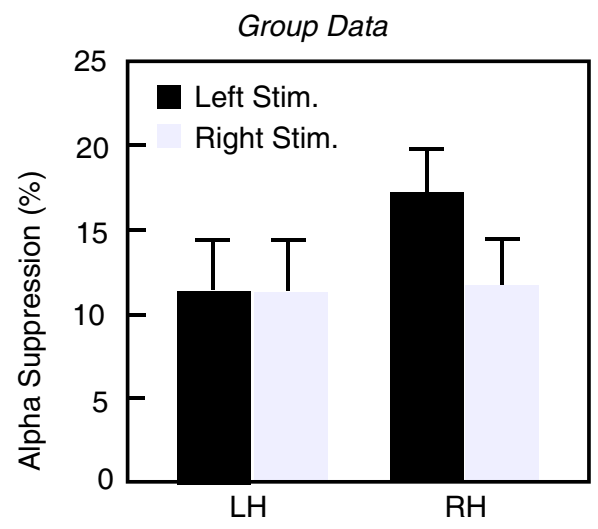

Individuals

\section{Participant 1}

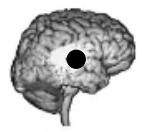

Participant 2

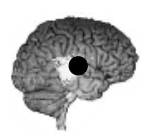

B

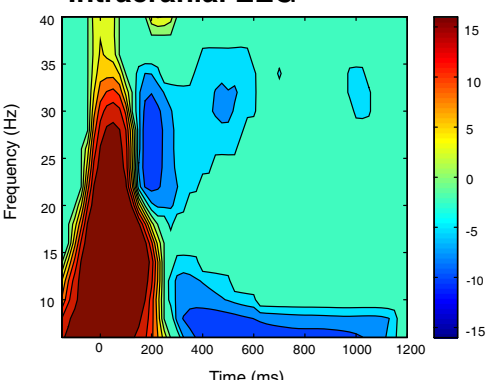

C
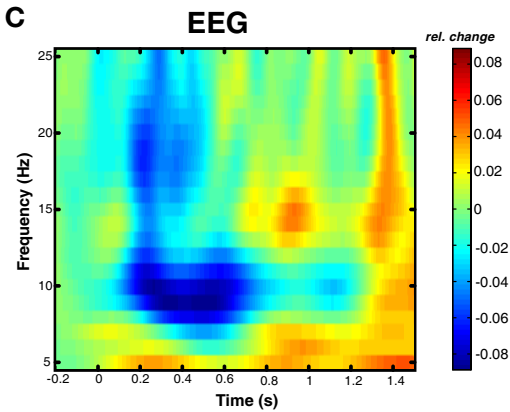

D

MEG

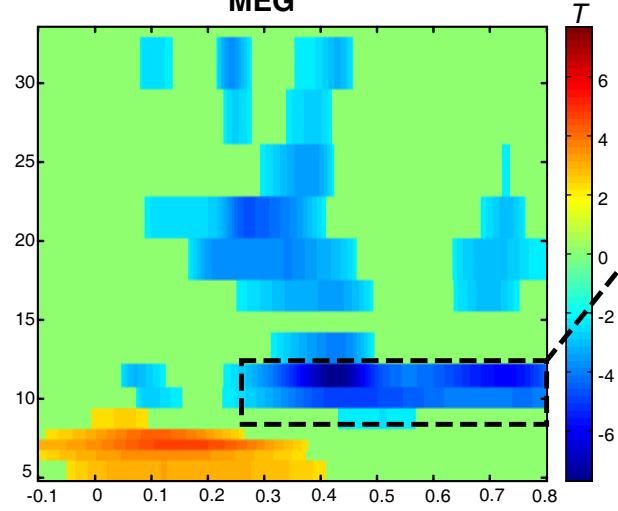

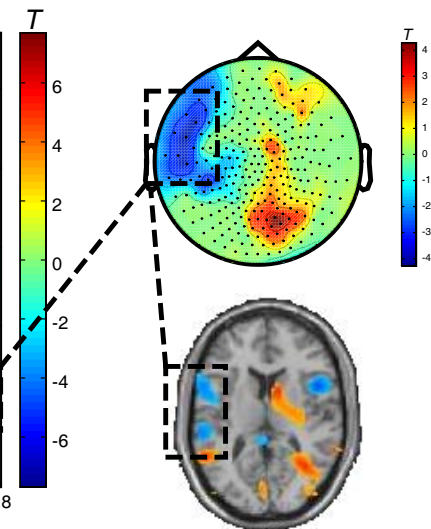

FIGURE 1 | Exemplary data illustrating the feasibility of recording alpha-like dynamics from auditory cortex. (A) Depicts an adaptation of data from the original experiment by Lehtelä et al. (1997) showing for the first time alpha suppression $(6.5-9 \mathrm{~Hz}$ ) at temporal MEG planar gradient sensors during sound presentation. In two individuals (right panel), the alpha desynchronization was localized to superior temporal regions overlapping with the generators of the $\mathrm{N} 1 \mathrm{~m}$ (here indicated as black circle; note that this circle has been exaggerated for better visibility). (B) Single-subject data from an sEEG experiment. The depicted electrode recorded activity from primary auditory cortex, showing desynchronization between 5 and $10 \mathrm{~Hz}$ starting $\sim 300 \mathrm{~ms}$ post-stimulus onset. Potentially earlier desynchronizations are masked by the strong evoked response within the first $200 \mathrm{~ms}$. Slightly higher alpha desynchronizations can be observed from group data in an (C) EEG experiment (time-frequency plot depicts an auditory cortical "virtual sensor" and a (D) MEG experiment. In the latter experiment a tone was presented to the right ear and post-stimulus decreases between 10 and $12 \mathrm{~Hz}$ are observable over left temporal sensors. These desynchronizations are mainly localized in left superior temporal regions including auditory cortex. Note that neither the sensor topography nor the source solution suggests a visual or sensorimotor contributions. other ear. Depending on the condition, the preceding cue indicated with 75 or $50 \%$ probability the location of the target (for auditory steady-state response results see Weisz et al., 2011). Source localization alpha desynchronization mainly indicate generators in left superior temporal gyrus including auditory cortex. Importantly, despite being a spatial attention task it is clearly observable that the auditory alpha desynchronization is not an epiphenomenon of visual cortex alpha desynchronization (e.g., "bleeding-in” of visual 
cortex activations). On the contrary, sensory topography as well as source results in Figure 1D point to a visual cortical increase of alpha, particularly pronounced ipsilaterally to the cued side (see also Foxe et al., 1998; Fu et al., 2001). This differential modulation of auditory cortical and visual cortical alpha activity is a very strong argument for separate processes in the respective sensory regions.

Overall, this section serves to establish that an auditory alpha rhythm exists that is independent from the other sensory modalities and that it can be measured even using non-invasive tools. It is also interesting to note that all data in Figure $\mathbf{1}$ also exhibit (in general more brief) desynchronizations in the beta range, indicating that beta activity may also constitute a resting state activity of the auditory cortex (this however exceeds the scope of the present review). At this stage it is not possible to give a general recommendation whether MEG or EEG is the more suitable tool to study auditory alpha activity. From the neuroanatomy, with large parts of the auditory cortex lying deep in the lateral sulcus and sources being mostly tangentially oriented to the surface, a superiority of MEG would be expected. However, there is a lack of a systematic investigation of this issue (e.g., identical paradigms used in EEG and MEG, ideally in separate recording sessions, as in our experience the simultaneous measurement of EEG often deteriorates the MEG signal). The overall measurability of auditory alpha - also using EEG - however does not answer questions with regard to its functional relevance. This will be the goal of the following sections.

\section{ABNORMAL SPONTANEOUS ACTIVITY IN TINNITUS BASIC PATTERNS OF TINNITUS-RELATED SPONTANEOUS ACTIVITY}

As pointed out in the introduction inhibitory mechanisms are a crucial ingredient for shaping adequate cortical functions (Buzsaki et al., 2007). More and more data from cognitive neuroscience (see below and other contributions in this special edition) suggest that the alpha rhythm may be the macroscopically recordable correlate of these inhibitory mechanisms. However, these experiments usually relate transient changes of alpha activity with well-defined stimuli and/or behavioral measures, i.e., they study short-term modulations of the E-I balance. This does not directly tackle the issue why under "rest" alpha-like oscillations should be the most dominant rhythm of the brain, whether it serves a function at all or just reflects "idling." Keeping up a "resting state" brain is metabolically very expensive (Gusnard et al., 2001), making it in pragmatic terms difficult to accept that the spontaneously expressed rhythmic activity should indeed reflect an idling activity. In the past (Weisz et al., 2007a) we have proposed that the dominant alpha activity at rest is functionally related to ongoing inhibitory mechanisms that prevent cell assemblies from spontaneous synchronization, which on a behavioral level could be associated with phantom perceptions.

One example for this notion that we have studied extensively in recent years is tinnitus. Tinnitus describes an audiological symptom in which a sound is perceived for which an objective environmental or bodily source cannot be identified. Phenomenologically, the perceptions are usually characterized by "simple" sounds such as pure tones (tonal tinnitus) or narrow-band noise. This condition most frequently follows a cochlear damage, e.g., after a noise trauma, sudden hearing loss or in the course of presbyacusis (age-related hearing loss). In the vast majority of cases psychiatric disorders (e.g., schizophrenia) can be excluded as a cause for the phantom percept.
In fact, almost everyone has experienced a tinnitus sensation at some point in their life, e.g., after attending loud concerts, etc., even though this sensation normally vanishes after a couple of hours to days. Also, it is very unlikely that the perception has its physical origins in an enhanced firing rate in the peripheral auditory system such as the hearing nerve (e.g., transection of the hearing nerve does not remove the tinnitus sensation in most cases; see Eggermont and Roberts, 2004 for a review). This poses scientifically a challenging issue: How can an overall healthy brain (assuming that anybody can perceive tinnitus under the "right" conditions) create a conscious auditory perception purely out of intrinsic, i.e., spontaneous, brain activity? An answer to this question has widespread implications for a better understanding of the neural correlates of consciousness (NCC; Crick and Koch, 2003) in general.

If a phantom percept like tinnitus can be viewed as a "normal" conscious perception minus a corresponding sensory input, then following current notions on the NCC (Dehaene et al., 2006; Lamme, 2006) at least two elements need to be involved: (1) The activation of specific sensory (in this case auditory) regions that are responsible for a specific feature of sensory experience. Such regions have been coined "essential nodes" (Zeki and Bartels, 1999) to note, that if this specific region is, e.g., destroyed then the subjective experience for this feature will disappear as well as in the case of agnosias. (2) The activation of an essential node does not take place in isolation but needs to efficiently transfer its processed information to higher-order brain regions (mostly frontoparietal systems) involved in attention and or consciousness ("global workspace"; Dehaene et al., 2006). The latter point concerns aspects of interconnectivity between distributed brain regions and will not be a matter of the current review (but to some extent relevant when discussing neurofeedback; see below). Here we will focus on the aspect that tinnitus requires an enhanced activation of the auditory cortex, which without a doubt is an important essential node for auditory perception.

The most direct evidence that tinnitus-enabling conditions lead to altered spontaneous activity in auditory regions has been gained from animal studies. In diverse species (e.g., cats, rats, chinchillas, etc.) and experimental conditions (e.g., noise trauma, salicylate, etc.) enhanced spontaneous firing could be recorded from multiple structures along the auditory pathway such as the dorsal cochlear nucleus, the inferior colliculus, primary and secondary auditory cortex (see for Eggermont and Roberts, 2004 a review). In a very important study investigating the immediate (i.e., within hours) changes in neuronal activity in primary auditory cortex of cats following noise trauma, Norena and Eggermont (2003) were able to show that increases of synchronized firing within deprived regions of the tonotopic map (i.e., frequency ranges affected by hearing loss) increase prior to increases in firing rate. Since tinnitus sensation usually develops rapidly following a noise trauma, the authors speculate that changes in synchronized firing could be more relevant for the actual tinnitus sensation than firing rate changes. This result has implications for human tinnitus studies, since non-invasive neuroelectric methods require the synchronization of a large population of neurons in order to be detectable at the head surface. With regards to the underlying mechanisms leading to the enhanced firing/synchrony, the majority of model frameworks based on animal data assume some downregulation of 
inhibition (Eggermont and Roberts, 2004) in deafferented regions of the auditory system to be responsible. Despite being a plausible hypothesis, to our knowledge direct evidence for an abnormal behavior in inhibitory neurons is missing up to date.

Investigation of human spontaneous brain activity in tinnitus has been more scarce and usually investigates altered spectral power in diverse frequency bands of the ongoing MEG/EEG. The first study was presented by our group and compared $5 \mathrm{~min}$ of resting MEG of chronic tinnitus sufferers to that of normal hearing controls (Weisz et al., 2005). The principal finding was an altered pattern of spontaneous activity in the lower frequency range with an increase of slow-wave (delta-theta; see also Moazami-Goudarzi et al., 2010) activity and a concomitant decrease of alpha power (see Figure 2A). This abnormal pattern was particularly pronounced over temporal regions and could be due to deviant ongoing brain activity in auditory regions of tinnitus participants. Our primary interest at the time of the study concerned the enhanced slowwave activity which frequently develops in deafferented neuronal tissue (Llinas et al., 1999). However, in the light of the increasing evidence from cognitive neuroscience relating alpha band activity to functional inhibition, it is tempting to assume that the decreased alpha activity in temporal regions may be a macroscopical neuronal signature of a reduced ongoing inhibition as hypothesized previously to be a crucial element for the emergence of tinnitus. Two pieces of evidence are further suggestive for this idea: Firstly alpha decreases in auditory cortex naturally accompany presentation of external sounds (see above). Since the crucial criterium for tinnitus is that an external sound cannot be identified accounting for the conscious perception, this could imply that the observed alpha reduction in tinnitus could represent a sort of "desynchronization" related to an internal sound. This would mean that apart of the external stimulus, the neuronal correlates of the sound perception are otherwise identical. Secondly, apart of changes in the lower frequencies we were also able to identify increases in the gamma frequency range. These increases are chronically present in tinnitus and are also modulated by slow-wave activity (Weisz et al., 2007b). Even though not a consistently reported finding, auditory cortical gamma activity has been associated to tinnitus laterality and/or intensity in some studies (Weisz et al., 2007b; van der Loo et al., 2009). Importantly, Lorenz et al. (2009; see Figure 2B) reported an inverse relationship between alpha and gamma activity calculated for sources seeded in auditory regions. The inverse relationship was present for tinnitus and control participants, however the regression slope was steeper in the tinnitus group, which was driven by the presence of an increased amount of participants who exhibited strong gamma activity alongside pronounced reductions of alpha band activity.

Based on our findings and notions from the animal literature we have proposed a model framework entitled "Synchronization by Loss of Inhibition Model” (SLIM), which summarizes our current notions on tinnitus. In the original version we assumed tinnitus to originate from a reduced afferent input in frequency regions affected by a peripheral damage. We postulated that this should lead to an overall reduced neuronal activity within the deprived region affecting equally excitatory as well as inhibitory neurons, resulting in a hyperpolarization. It has been shown previously that hyperpolarized neurons fall into a slow-frequency bursting mode (Llinas et al., 1999) which could account for the enhanced delta to theta activity in tinnitus sufferers (Jeanmonod et al., 1996; Weisz et al., 2005; Moazami-Goudarzi et al., 2010). Crucially, we hypothesized that in a circumscribed region of tonotopically organized auditory fields, downregulation of inhibitory drive is strong enough and excitatory neurons are not hyperpolarized sufficiently, that the latter "spontaneously" synchronize their activity. While, in contrast, a loss of inhibition may be reflected in reduced alpha activity, we assumed that "spontaneous synchronization" may be expressed by enhanced gamma band activity. The inverse relationship between resting-state alpha and gamma point in this direction (Lorenz et al., 2009), however the anticorrelated behavior of alpha and gamma frequently seen in sensory cortices following external stimulation is also supportive of this basic idea: In almost all cases in which gamma increases are observed in sensory and motor cortex a concomitant decrease of alpha power can be observed (see e.g., Figure I in Jensen et al., 2007, for exemplary data; however a

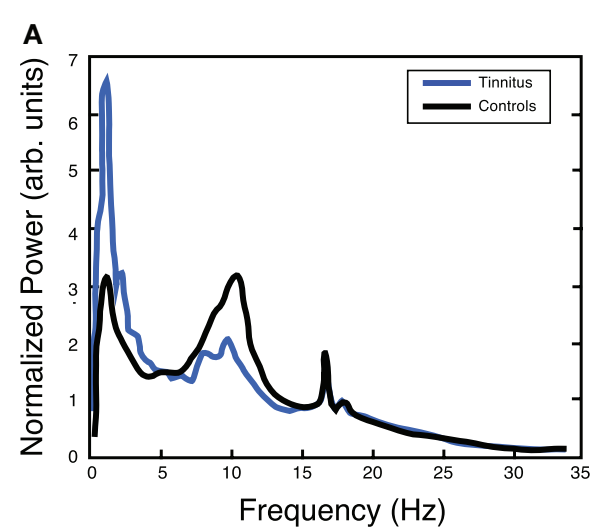

FIGURE 2 | (A) Chronic tinnitus is characterized by marked changes in ongoing ("resting") oscillatory brain activity, including a reduced alpha peak as compared to normal hearing controls with a focus over temporal regions (adapted from Weisz et al., 2005). (B) The inverse relationship between ongoing alpha and gamma activity

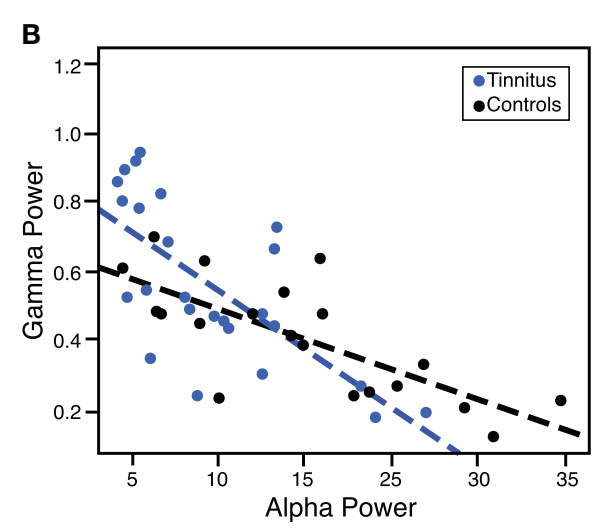

in temporal areas suggests that mechanisms generating alpha oscillations may be crucially involved in regulating the $\mathrm{E}-\mathrm{I}$ balance. Note that in the tinnitus group this relationship is steeper due to a higher proportion of participants with low alpha and high gamma levels (adapted from Lorenz et al., 2009). 
decrease of alpha accompanied by gamma increases is not always evident, perhaps due to the capacity of the paradigm and noninvasive method to resolve low amplitude gamma band activity). If spontaneous synchronization is sufficiently strong (potentially amplified by top-down influences; see below), this activity will be passed on to downstream cortical regions involved in conscious perception (Schlee et al., 2009b). The basic idea of our tinnitus model is summarized in Figure 3. It has to be emphasized that our framework is descriptive does not contain a detailed assumption of how this inverse relationship between alpha (putatively reflecting "inhibition") and gamma (putatively reflecting "synchronized firing") comes about. Based on cognitive neuroscientific works different ideas have been proposed based on the basic idea that within a cycle of an oscillation excitability is modulated, opening and closing windows of opportunity for neurons to synchronize their firing. Jensen et al. (2007), e.g., argues that an ongoing alpha activity is disadvantageous for precise synchronization of neuronal firing, thereby limiting the postsynaptic impacts of respective alpha-activated cell assembly. Gamma activity on the other hand induces short time windows in which neuronal firing can synchronize, thereby increasing the postsynaptic impact of the gammaactivated cell assembly. A somewhat different idea was forwarded by Klimesch et al. (2007) suggesting the alpha rhythm is explicitly caused by inhibitory interneurons and that different phases of the alpha reflect a stronger or weaker impact of these neurons on tonically excited excitatory neurons. In cases of strong alpha activity (thus reflecting strong activity of inhibitory neurons), synchronization of neuronal activity will change from a tonic into a rhythmic modus see (Schroeder and Lakatos, 2009 for a similar idea).

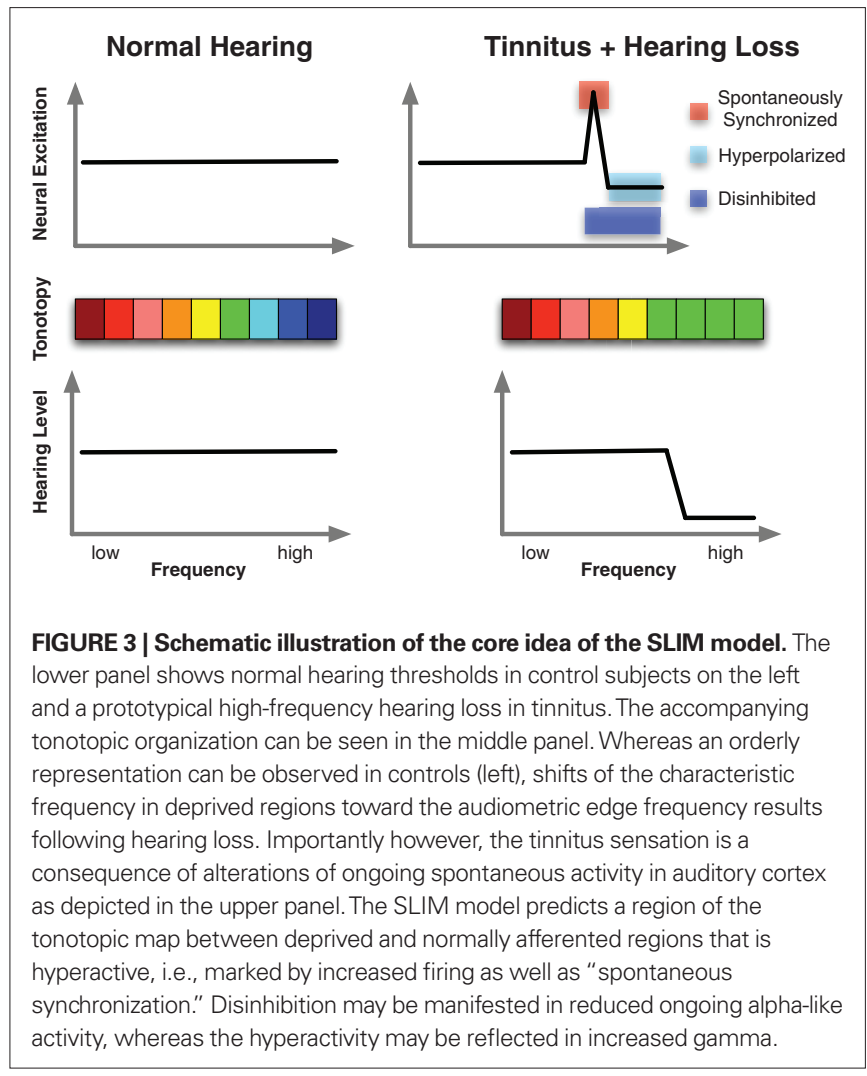

Importantly in both frameworks, periods of low alpha would go along with enhanced and sustained synchronization of neuronal activity, which as a consequence has a greater postsynaptic impact on downstream neuronal assemblies.

Recently, we conducted a study (Ortmann et al., 2011) that challenges some of the basic ideas set out in the original model. In this study we investigated transient tinnitus in rock musicians following band giving us the potential opportunity to study the macroscopic neuronal processes that immediately accompany tinnitus sensation. The consistent finding of the study was that compared to another MEG measurement without tinnitus (appointments separated by at least 1 week), band practice was followed by strong increases of gamma band activity in right auditory cortex (in 13/14 participants). However, we did not find any evidence for a decrease of alpha or increase of slow-wave activity in auditory cortex. At this stage it is premature to definitely decide between different possible interpretations: For example, it is also conceivable that tinnitus-related activity is spatially very restricted, making it challenging to monitor them non-invasively. Not mutually exclusive to that, the transient and chronic tinnitus may involve partially different neurophysiological mechanisms either from the outset or developing over time, leading to an important question: Why does chronic tinnitus develop only in a minority of cases? One hypothesis directly following from the framework presented in this review, would be that severe distortions of the E-I balance might be necessary for tinnitus to become chronic, maintaining the spontaneously hypersynchronized state. This imbalance could be reflected in reduced alpha-like activity in relevant regions of the auditory cortex, however at this stage the idea is very speculative.

To summarize, we think that chronic tinnitus provides a good clinical case for ongoing alpha-like activity serving an important function in auditory cortex. Beyond the tinnitus field, ongoing (spontaneous) alpha activity in sensory and motor regions, sometimes described as "idling" activity, could functionally be the neurophysiological correlate of an ongoing mechanism of active suppression of "spontaneous synchronization." Since phantom percepts such as tinnitus (but potentially also phantom limb pain) may be a consequence of deficiencies of ongoing inhibitory mechanisms, leading to spontaneous synchronization, we argue that effective treatments re-establishing/normalizing the E-I balance will have an impact on ongoing alpha activity. Several approaches are conceivable, reaching from pharmacological (Elgoyhen and Langguth, 2010) to brain stimulation techniques (Kleinjung et al., 2008). Another possibility however is neurofeedback, which will be introduced in the subsequent section.

\section{NEUROFEEDBACK TREATMENT OF TINNITUS}

The neuroscience of tinnitus is a fascinating field, since it opens up the possibility of studying the NCCs in a "pure" form, i.e., without concomitant activation by a physical stimulus. Yet delving into the neurophysiological mechanisms leading to this phantom percept is of outstanding clinical relevance as well. It is estimated that approximately $10-15 \%$ of the population in industrialized countries are affected by chronic tinnitus (Eggermont and Roberts, 2004). In approximately $1-3 \%$ of the population tinnitus is so distressing that it causes severe impairments in the quality of life and therewith related high socioeconomic costs (e.g., missing working hours or 
early retirement). Unfortunately at the current stage there is no therapy that can reliably eliminate the tinnitus sensation. This is definitely partly due to the limitations of the current approaches in fully tackling the generating mechanisms (see e.g., Lorenz et al., 2010 for a study on rTMS) but also perhaps due to a greater complexity than the idea of a stable and invariant generating mechanism (see above and also Schlee et al., 2009a for a study on tinnitus duration and associated neurophysiological changes). Nevertheless, the field is progressing with some neurophysiologically well-founded therapy approaches that show modest success rates. In general the common theme of these therapy approaches is that they aim to normalize spontaneous activity and/or tonotopic organization in the central auditory system. Two different types of approaches can be differentiated: (a) bottom-up and (b) top-down based therapies. Bottom-up based therapies include, e.g., sound-stimulation therapies (e.g., "notched music," Okamoto et al., 2010; or "coordinated reset," Hauptmann and Tass, 2010) or direct stimulation of auditory cortex, e.g., by rTMS (Kleinjung et al., 2008) or implanted electrodes (De Ridder et al., 2007). Top-down based therapies assume that higher-order mechanisms (such as attention; see below) can influence excitability in auditory cortical regions (see Andersson et al., 2006 for a fMRI study and tinnitus). One of these approaches is neurofeedback that will be the topic of the present section.

Neurofeedback is an approach of treating diverse maladies, that has existed for about 40 years already (Sterman and Friar, 1972) and is theoretically founded on operant learning, i.e., to increase "correct" behavior through reinforcement. In this case "correct" behavior describes what the experimenter assumes is a neurophysiologically "healthy" pattern and reinforcement implies a feedback to the patient with regards whether she/he is expressing this desired pattern (including a "reward" when certain goals are reached). The overall goal of neurofeedback is that the participant learns to control abnormal neurophysiological processes purely by intrinsic means (i.e., mental strategies). Basically three elements are of outstanding importance for neurofeedback to work: (1) a good hypothesis of what may be the crucial abnormal neurophysiological signature of a distinct disease, (2) good equipment and signal processing routines to monitor the neurophysiological feature of interest in "realtime," and (3) an "immediate" and intuitive feedback to the participant. The latter two aspects are mainly computational issues for which great advances have been made over the years (e.g., wide accessibility of powerful signal processing routines, fast multi-core computers, high-density EEG recordings etc.). Thus in our opinion the main challenge for neurofeedback is actually of conceptual nature, i.e., defining the core neurophysiological mechanisms of a disorder and identifying its macroscopic - non-invasively measurable - expressions.

Based on our MEG studies (see above) we conducted two different approaches mainly targeted at re-establishing normal alphalike activity in auditory cortex. Conceptually, the approaches are founded on the idea introduced above that alpha-like activity reflects an ongoing inhibitory process suppressing spontaneous synchronization (see above). The first approach, which we will call "Alpha-Delta Training" subsequently, seeked to normalize the pattern of spontaneous EEG activity by aiming to enhance alpha and reduce delta activity. A previous study yielded promising results $(\sim 20 \%$ reduction of tinnitus distress; Dohrmann et al., 2007$)$ using only four frontocentral EEG electrodes and a simple ratio measure (i.e., alpha/delta; one-dimensional feedback). Motivated by these findings we attempted to overcome certain issues in a modified Alpha-Delta Training $(n=10)$. The first issue concerned the feedback from four frontocentral electrodes, which makes it highly unlikely that ongoing oscillatory activity from auditory cortex was trained, but probably a mixture from diverse brain regions. The second issue concerned the interpretational limitations of a ratio, since increases could mean increases of alpha, reductions of delta or both (the limitation was due to the proprietary software used). In order to tackle these issues, we developed our own software framework for online EEG (ConSole; Hartmann et al., 2011). The first improvement was the recording from a higher number of electrodes (32 channels) and the use of a source montage, modeling ongoing brain activity from temporal brain regions. This measure reduces to some extent the problems of the mixture of different signal contributions encountered in sensor space. The second change was the use of a two-dimensional feedback, in which alpha and delta power were two different axes on a computer screen. The task of the participant was to move the ball as far as possible to the upper right corner, indicating "high alpha" and "low delta" (see Figure 4A). The second training approach $(n=7)$, which we will call "Desynchronization Suppression Training" subsequently, used exactly the same hardware and source projection method. However in this approach we used sounds of $5 \mathrm{~s}$ duration, which consisted of a band-passed noised containing input at frequencies of the "tinnitus spectrum" (Norena et al., 2002). The task of the participant was to increase alpha activity as much as possible during sound stimulation (Figure 4B), i.e., to suppress the "natural" tendency of alpha desynchronization (see above). The rationale of the Desynchronization Suppression Training was that the participant learns to decrease excitability to a sound spectrally similar to the own tinnitus sensation and to transfer successful strategies into every day life.

Despite the different procedures underlying the two neurofeedback approaches some common results emerged. For both trainings alpha and delta changes were operationalized by calculating the ratio between pre-training and post-training power (pre/post). In both trainings delta was only marginally modulated, even in the training in which explicit feedback was given [Alpha-Delta Training (mean \pm SE): $0.93 \pm 0.07$; Desynchronization Suppression Training ${ }^{1}: 1.17 \pm 0.33$ ] and will not be further reported in this article. Contrary to slow-wave activity, participants were overall very successful in enhancing alpha-like activity from temporal sources. The increases in the Alpha-Delta Training reached almost $80 \%$ and were larger than $50 \%$ in the Desynchronization Suppression Training independent whether a sound was being processed or not (see Figure 4C). This means that our procedures were successful in globally increasing alpha activity in temporal brain regions. Furthermore, on average tinnitus-related distress (assessed using the "Tinnitusfragebogen"; Goebel and Hiller, 1994 ) was decreased on average by $\sim 27 \%$ (Alpha-Delta Training: 28\%; Desynchronization Suppression Training: $25 \%$; $t_{15}=-0.16$, $p=0.87$ ). This overall reduction (see Figure 4D) was statistically

${ }^{1}$ The pattern was similar for a 5 -s pre-stimulus and the 5 -s post-stimulus period and therefore averaged in this report. 

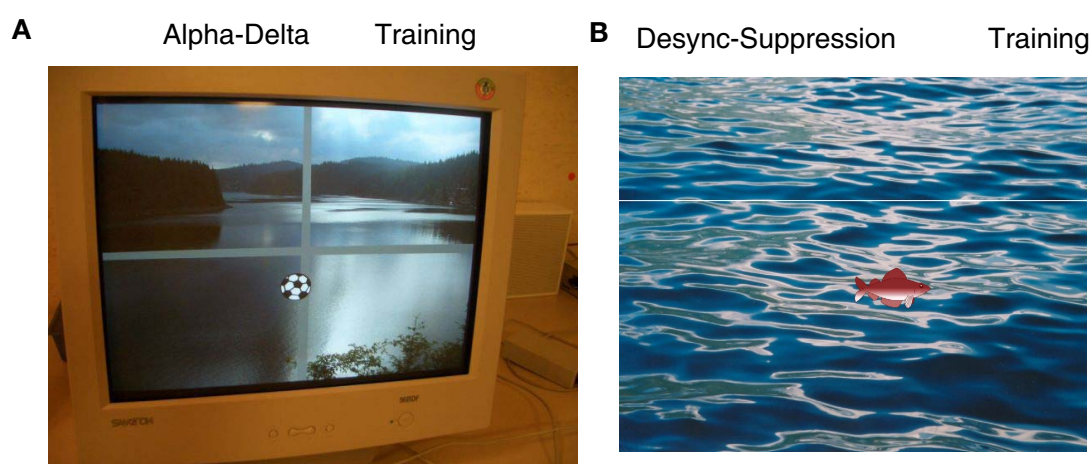

C

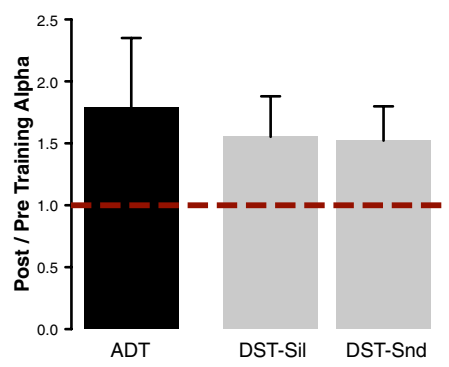

FIGURE 4 | Demonstration of the feedback for the participants of our neurofeedback training. (A) In the Alpha-Delta Training alpha and delta power were fed back by movements of a football in 2D. Movements of the ball into the right upper quadrant was desired, marking increases of alpha with concomitant decreases of delta in temporal sources. (B) In the

Desynchronization Suppression Training decreases of alpha in temporal sources upon sound stimulation were visualized by a fish moving downwards. The goal

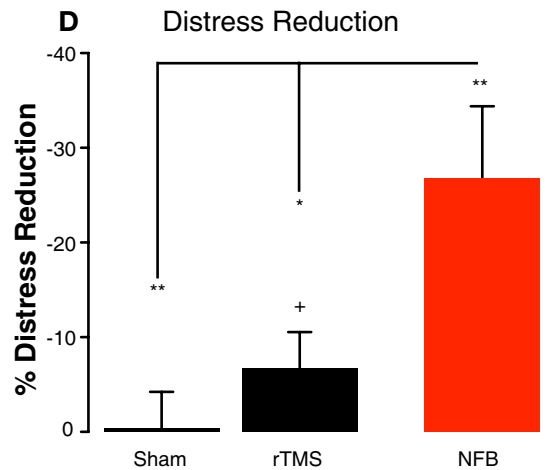

of the participant was to move the fish upwards indicating a suppression of desynchronization. (C) Both neurofeedback trainings were successful on average in increasing alpha-like activity in temporal brain regions. (D) Training success went along with highly significant reductions of tinnitus distress (red bar). Distress reductions are significantly stronger as compared to a shamrTMS group as well as compared to a $1-\mathrm{Hz}$ rTMS group (targeting auditory cortex).

putative hyperexcitation in auditory cortex. Accordingly our rTMS treatment ( $n=24$; pooling data from Konstanz and Regensburg) included 10 sessions of $1 \mathrm{~Hz}$ rTMS of auditory cortex and 10 sessions of sham stimulation (coil tilted by $45^{\circ}$ ), counterbalanced across participants. In order to maintain comparability across treatments, we used tinnitus distress immediately before the first and directly following the last treatment session. As observable from Figure 4D distress was virtually unchanged after sham treatment (one-sided test: $t_{23}=-0.09, p=0.92$ ) and slightly but significantly reduced ( $~ 7 \%$; one-sided test: $\left.t_{23}=-1.75, p=0.04\right)$ following real $1 \mathrm{~Hz}$ rTMS. Compared to the rTMS results, the $27 \%$ reduction observed following neurofeedback therefore appears meaningful. This impression is statistically confirmed: distress reduction following neurofeedback is significantly greater than sham rTMS (two-sided test: $t_{23}=-3.34, p=0.002$ ) as well $1 \mathrm{~Hz}$ rTMS (two sided test: $\left.t_{23}=-2.55, p=0.01\right)$. While the effect compared to sham treatment is intuitive, the stronger distress reduction as compared to rTMS is not trivial and leaves several open questions. The main issue is whether the stronger distress reduction stems from a greater influence of neurofeedback on auditory cortical activity or on widespread networks. Due to the very basic source projection approach and low number of electrodes (32 channels), the second answer appears more appropriate, upon further empirical evidence can 
be gathered using more advanced source modeling and a higher number of electrodes (or alternatively MEG-neurofeedback). Furthermore it will be relevant to scrutinize whether neurofeedback actually indeed successfully modulates tinnitus-specific brain activity or simply is more successful than rTMS in tackling non-specific processes that influence distress scores. An answer to this question would require a neurofeedback-specific placebo condition.

Despite these shortcomings, the reported findings encourage further developments in neurofeedback as a treatment option for chronic tinnitus. Our results suggest that reduced temporal alpha activity can serve as a relevant neurophysiological marker of tinnitus, and deeper insights into how auditory alpha-like oscillations reflect the E-I balance of auditory cortical cell assemblies will open up new avenues for the treatment of this distressing disorder.

\section{COGNITIVE MODULATIONS OF ALPHA}

In the latter two sections we have (a) demonstrated that an auditory alpha-like rhythm exists and (b) that disturbances to this resting rhythm may be a fundamental element for developing tinnitus, indicating that the auditory alpha rhythm may represent the E-I balance within the respective region, similar to the visual and sensori- and motor cortices. Yet the latter example illustrates potential behavioral consequences of a permanent departure of normal resting state activity. In everyday life however we are confronted with the need to adapt E-I balance in sensory and motor regions on a short time scale (transient modulations). Common examples are, e.g., selective attention during which processing resources are specifically allocated to certain aspects in our environment while other aspects are ignored or working memory which describes the capacity to hold certain information in mind for (relatively) short periods of time. For both cognitive abilities to work properly excitability in task-relevant areas needs to be enhanced, whereas regions that process competing/task-irrelevant information need to be disengaged. If alpha oscillations indeed represent the E-I balance within (at least sensory) cortical regions, then task-related short-term modulations of excitability should be mirrored in concomitant modulations in regionally specific alpha power, i.e., relative decreased alpha power in task-relevant regions and increases in regions processing distracting information. In the visual domain this notion has found extensive support in recent years (Foxe et al., 1998; Worden et al., 2000; Fu et al., 2001; Thut et al., 2006; Meeuwissen et al., 2011), however for the auditory modality this evidence is still largely lacking (see van Dijk et al., 2010 for a recent demonstration in auditory working memory). In this section we will introduce some of the running works from our labs to resolve this issue, by illustrating their rationale as well as representative single-subject data. The first section will address the issue of auditory alpha and attention/ anticipation, whereas the second section will introduce concepts of studying alpha in a special cognitive domain linked to the auditory system, namely speech comprehension.

\section{AUDITORY ALPHA AND ATTENTION}

In cognitive science attention is not treated as a unitary phenomenon and is generally viewed as the allocation of processing resources that can be driven by bottom-up and/or top-down mechanisms. The former refers to neuronal processes triggered by distinct features of a stimulus, whereas the latter, also termed selective attention, refers to endogenous processes such as the pursuit of internal goals or anticipation. Interestingly, scientific investigation of selective attention actually started in the 1950s in the auditory domain when Cherry (1953) investigated the so-called "cocktail party problem," i.e., the capability of listeners to monitor a distinct stream of conversation while ignoring irrelevant background noise. Unfortunately, cognitive neuroscience and particularly the branch interested in the relationship between alpha and attention, has thereafter mainly focused on the visual modality. This may also be partly due to the long uncertainty, as to whether an auditory alpha rhythm existed that is independent of those of other modalities. From the studies in the visual modality, which usually employ a variant of a Posner-like cue-target paradigm (Posner and Petersen, 1990), the following important insights have been gained: (1) Alpha power in visual cortical regions processing to-be-attended visual information decreases, indicating an enhanced excitability of these regions (Thut et al., 2006). (2) Alpha power in visual cortical regions processing to-be-ignored visual information (requiring the presence of distracters in the paradigm) increases, indicating decreased excitability of these regions (Worden et al., 2000; Rihs et al., 2007). Interestingly, this pattern of posterior alpha increase has been also reported when attention is cued to the auditory modality, putatively reflecting an active disengagement of attentional resources from the visual modality (Foxe et al., 1998; Fu et al., 2001). (3) These processes take place prior to the presentation of the target, implying that alpha modulations also reflect preparatory processes of tuning the E-I balance in visual cortical tissue to optimally process the anticipated target. Interestingly, a recent study (Capotosto et al., 2009) demonstrated that these preparatory alpha modulations can be severely perturbed and even abolished by applying TMS either to the intraparietal sulcus (IPS) or the frontal eye field (FEF). For the auditory domain this is interesting also, since a recent study by Smith et al. (2009) showed that rTMS applied to the FEFs interferes with auditory spatial localization using a visual cue: The behavioral costs of being invalidly cued were considerably reduced. Taken together, one could derive the hypothesis that selective attention functions on a higher level by modality independent mechanisms, modulating the E-I balance in lower level sensory regions. However for this assumption to apply it has to be at first established that auditory cortical alpha is indeed sensitive to attentional modulations.

\section{Auditory cortical alpha activity reflects direction of auditory spatial attention}

In a previous section, we had presented additional evidence for the existence of an auditory alpha-like rhythm that adds favorably to the previous literature (e.g., Lehtelä et al., 1997). Currently the Konstanz laboratory is running a series of studies to investigate whether this ongoing rhythm can be modulated by selective attention. In a first study (Müller et al., submitted) we implemented an auditory spatial attention task in which participants had to indicate the presence of a target (10\%; changes in amplitude modulation frequency of a tone) on a visually cued ear ( $100 \%$ cue validity). Sounds were presented on both ears, therefore the task included the selective monitoring of an auditory channel while ignoring distracting information from the task-irrelevant ear. Following the offset of the sound, participants had to indicate via a right hand button press whether a target was present or not. The focus of our analysis was on pre-target 
alpha modulations which have been previously demonstrated in the visual modality (Thut et al., 2006). As expected, our main finding was an asymmetric modulation pattern in left and right auditory cortex depending on which ear was cued. Data from a single subject is depicted in Figure 5, showing (relative to a pre-cue baseline period) stronger alpha desynchronization at sensors contralateral to the visually cued ear. Beamformer source analysis indicates besides of motor preparatory activity, that this desynchronization pattern is generated in the auditory cortex. Interestingly (data not shown here), relevant periods of pre-target alpha desynchronization went along with specific changes of functional connectivity between auditory cortex and particularly FEF and IPS. Whereas auditory regions processing upcoming information from the attended ear were more strongly coupled to these regions, those processing information from the unattended ear exhibited decreased functional connectivity as compared to the pre-cue baseline. Despite the absence of a genuine alpha synchronization (i.e., increase relative to baseline), the results are supportive of a general notion forwarded by Jensen and Mazaheri (2010), that levels of alpha activity may gate information flow within distributed networks.

This study confirms that similar attentional alpha modulations can be observed in the auditory modality as in the visual modality and is also suggestive that similar higher-order regions (Capotosto et al., 2009; Smith et al., 2009) may be involved in modulating the E-I balance across sensory modalities. It is worth noting at this point that a lateralized pattern of pre-stimulus alpha does not have to be necessarily expected, since - even though a majority of nerve fibers cross at the level of the brainstem (the superior olivary complex being the first structure to receive information from the contralateral ear), thus producing a structurally contralateral representation similar to visual and somatosensory cortex - the auditory cortices of the two hemispheres appear to be functionally more specialized than the respective areas of the visual and somatosensory system. For

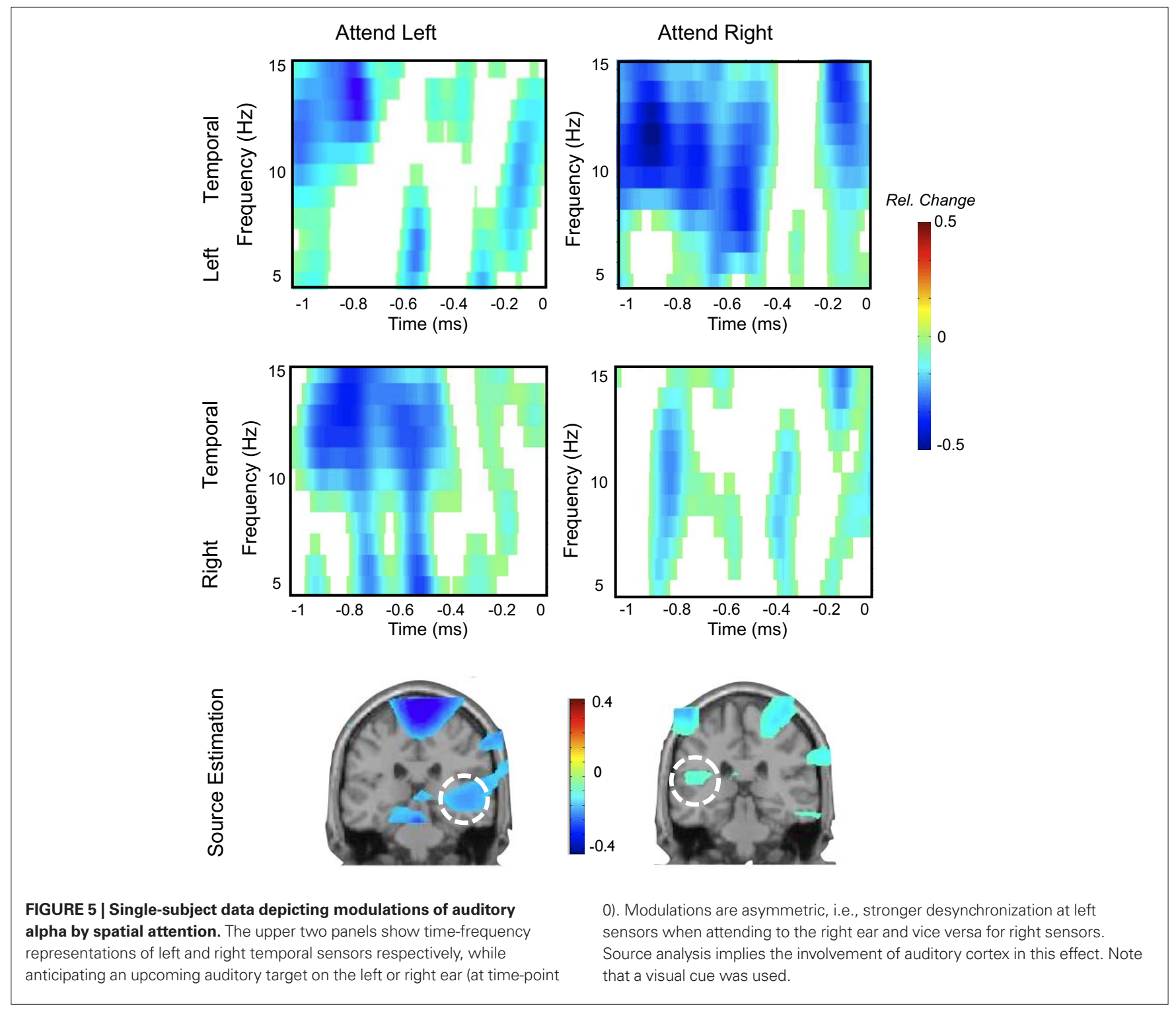


example speech (see below) and music appear to be dominantly processed by the left or right auditory cortex respectively (details go beyond the scope of this review; see Zatorre et al., 2002). Importantly in the context of this study is that spatial information appears to be preferentially processed by the right auditory cortex, which is involved in computations of the whole space, whereas the left auditory cortex is largely involved in processing the contralateral (i.e., right) space (Zatorre and Penhune, 2001; Spierer et al., 2009). It is possible that in the current experiment the lateralized pattern is favored by the presence of a distracting stimulus on the ear contralateral to the target, i.e., in the case of a distracter on the left ear it seems advantageous to downregulate activity in the right auditory cortex, thus influencing its normal bilateral involvement. In this experiment expectations were externally triggered by the use of visual cues. This leaves the question open whether auditory alpha activity is also sensitive to totally internally generated expectations.

\section{Illusory impression of perceiving a salient sound is reflected in stronger auditory cortical alpha desynchronization}

To tackle the issue whether auditory cortical alpha activity can reflect internally generated expectations, we employed a pseudo frequency discrimination task (Hartmann et al., submitted). Participants were trained to differentiate two tones slightly varying in pitch ("low" versus "high" tone). The feedback to the participant was that each time they heard the "high" tone, an aversive loud noise was played at the end of the 8-s stimulus (response given between 6 and $8 \mathrm{~s}$ ). By these means participants learned to associate the presence of a "high" tone with an aversive consequence (classical conditioning), thus making the "high" tone salient. In the real EEG experiment, we presented always the identical tone (not corresponding to the training tones) along with a low-intensity narrow-band noise. Participants were instructed, that this noise could make the frequency discrimination task extremely difficult. In order to promote changes in expectations, we pseudo-randomly manipulated the feedback to the participant using a procedure borrowed from Perruchet (1985). Indeed the sequence of feedback significantly influenced which tone the participant reported to have actually perceived (i.e., the more sounds were followed by a loud aversive noise on previous trials the less likely participants reported the presence of a "high" tone on the current trial). When comparing trials on which participants reported hearing a "high" tone as compared to those on which they reported hearing a "low" tone, the EEG showed stronger decreases of alpha activity in auditory cortex in the former condition (see Figure 6 for a single subject). The differentiation between the condition commenced with sound onset (i.e., no pre-stimulus effects) and built-up gradually to reach a peak around 2-4 s post-stimulus onset. This study shows that the perception of a more salient sound, is associated with a stronger alpha decrease in auditory cortex, putatively indicating the enhanced excitability of this region (Gross et al., 2007 for gamma in somatosensory modality). Since the physical features of the stimulus were identical on each trial, this implies that auditory cortical alpha activity faithfully monitors the trial-to-trial dynamics of internally generated expectations.

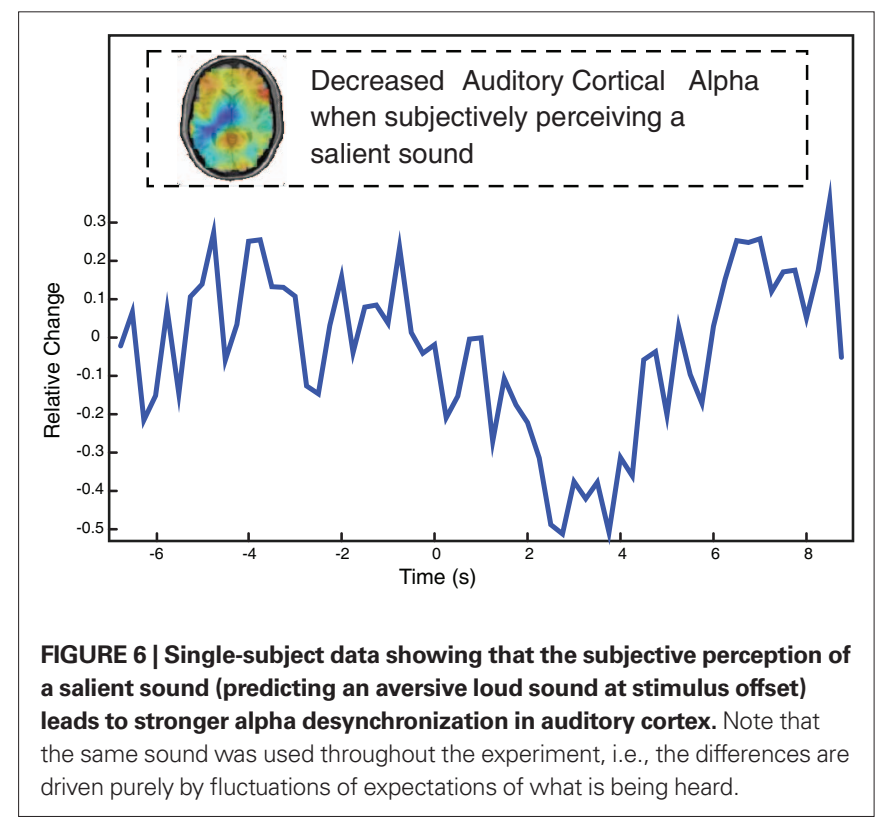

\section{AUDITORY ALPHA AND SPEECH PROCESSING}

As has become clear by now, our knowledge on auditory induced alpha-frequency perturbations and how they relate to cognitive functions is sparse. This is particularly true for the most behaviorally relevant and most sophisticated auditory percept humans are specialized in, that is, speech. This is unfortunate, as speech perception forms a prime case for studying cognitive mechanisms in the auditory domain - especially so under naturally adverse conditions or in degraded hearing. Also, brain physiological parameters are needed that would indicate successful comprehension or degree of effort that is required for comprehension - at best in the single participant rather than on a group level only.

What is the current evidence on changes in the alpha-frequency band during speech perception and comprehension? While experiments that target this question directly are sparse, some observations can be derived from two related threads of evidence: First, studies that were focused on language processing (mainly semantic and lexical processes) and used auditory presentation (e.g., Shahin et al., 2009); second, studies that have used speech stimuli primarily as a carrier or as a means to study related cognitive phenomena such as working memory or attention (e.g., Kerlin et al., 2010). Of these few studies, most do not focus on the alpha band, but many observe and report changes in this frequency range nevertheless.

As for the first group of speech/language-alpha studies, Bastiaansen and Hagoort (2006) have put forward that most alpha power changes reported in language comprehension studies can be traced back to the task manipulations employed rather than to the language stimuli directly. (Note that this conclusion, which has since not been considerably challenged, is based to large degrees on visually presented stimuli.) Even if so, however, it does not detract from alpha's potential relevance for the cycle of sensation-perception-comprehension in the auditory domain: Speech comprehension often becomes a taxing task, in particular when degraded signal quality or degraded hearing, or both, begin to 
affect the sensation and perception of the speech signal itself. These challenges closely link speech comprehension to more domaingeneral cognitive resources such as working memory, attention, and executive control. It is in this framework that more specific hypotheses for the function and response behavior of alpha activity in speech comprehension can be postulated:

\section{The magnitude of alpha suppression, as observed in response to linguistic stimuli, depends on sufficient signal quality}

Alpha suppression (compared to a reference or baseline period) has been observed in the aftermath of a word or other linguistic stimulus presentation (e.g., Klimesch et al., 2007; Shahin et al., 2009). A parsimonious explanation for these observations would be that the decrease in alpha power reflects, by relay, an increased level of cognitive processing (Jensen and Mazaheri, 2010), such as accessing the lexical meaning of an utterance. The extent to which these computations are allowed to take place should depend on the signal quality (such dependency on signal quality has been recently shown for semantic integration reflected in the auditory gamma band response to more complex linguistic stimuli; Obleser and Kotz, 2010).

To answer this question, we recently conducted an EEG study on auditory comprehension of words that had been degraded to various degrees using noise-band vocoding (Shannon et al., 1995; Scott et al., 2000; Obleser et al., 2008), a technique effectively reducing the spectral and temporal content of the speech signal to arbitrary degrees, and mimicking the input provided by a cochlear implant (Shannon et al., 1995). As outlined above, we were expecting to observe suppression of alpha power when presenting listeners with degraded audio signals of words that had sufficient level of acoustic detail to allow word recognition. Preliminary data from our labs, however, go beyond this and show a parametric dependence of the alpha suppression magnitude on acoustic detail present in the signal (Obleser and Weisz, 2010; Obleser and Weisz, in preparation):

The less amount of spectral detail (i.e., fast spectro-temporal change) was present in a stimulus, the less peri- and post-stimulus alpha power deviated from baseline. The same held true, to a weaker degree, for variations in the temporal detail (i.e., slow envelope change) of stimuli. The monotonic decrease in alpha power as a function of acoustic detail occurred first about $500 \mathrm{~ms}$ after a word's onset, that is, around a time when access of the word meaning became possible. (Speech, of course, unfolds over time and the information minimally required for identification accumulates). Interestingly, the topography of this effect varies somewhat between single subjects we so far analyzed. A group statistic showed the effect to be strongest at parietal scalp sites, and localized to the superior parietal cortex accordingly. The single subject shown here, however, shows this effect at left temporal scalp sites (Figure 7). Figure 7 shows the monotonic decline of alpha power as the signal quality improves (2- to 16-bands noise-vocoding; restoring more spectral detail of the speech signal and allowing for much improved intelligibility).

The variations of task should be taken particularly seriously in speech and language experiments. Interestingly, a recent replication of the experiment in two of the previous subjects, without any active rating task, showed the same parametric alpha power change (centered at $11 \mathrm{~Hz}$ around $1 \mathrm{~s}$ after stimulus onset) to localize all the more clearly to the temporal lobe when no active rating but only prompted button presses were required.

How should we interpret the decline in alpha power as a function of intelligibility of the signal? As argued above, the relative decline in alpha power is likely to reflect mental operations on the speech signal, thus, active cognitive processing. A post-hoc look into changes in Gamma power and the observed increase of Gamma power within the clusters identified as alpha suppression clusters is in favor of this interpretation (cf. Osipova et al., 2008). However, the lack of such alpha suppression relative to a no-stimulus baseline in severely degraded speech might as well reflect counteracting activity in neural oscillators which keep the alpha power high in order to gate language- and meaning-related areas. This argument also derives from the functional inhibition framework: On the one hand, relative increases of alpha power have been consistently reported during working memory retention

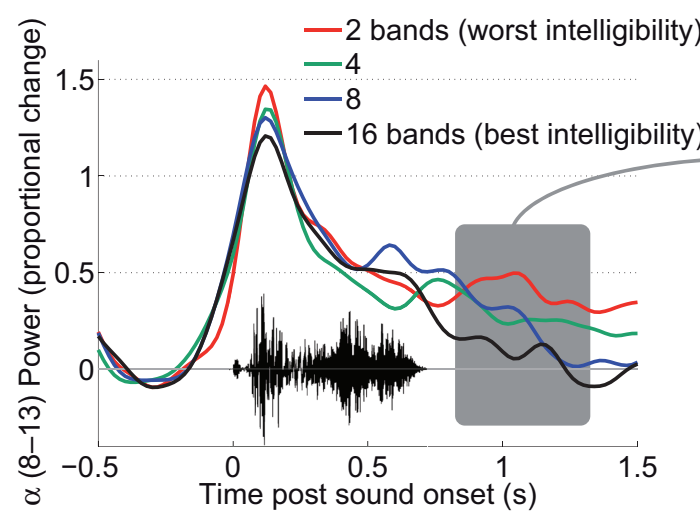

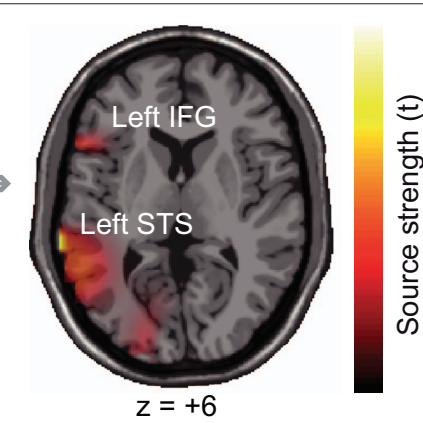

Parametric decrease in a power from 2 to 16 bands (single-trial beamformer statistics)
FIGURE 7 | Single-subject data on late alpha suppression in word comprehension (EEG) as a function of signal quality. The left panel shows the time course of alpha power. The parametric suppression of alpha power in this participant surfaces as a relative decline. A beamformer projection of the single trials and ensuing statistics show that, in this participant, languagerelated areas in the left superior temporal sulcus and left inferior frontal gyrus are likely sources of this parametric change in alpha power dependent on signal quality. 
(e.g., Jensen et al., 2002; for a version using auditory syllables, e.g., Leiberg et al., 2006), reflecting inhibitory control over items in memory (see Klimesch et al., 2007 for review). On the other hand, it has been argued before that listening to degraded speech also taxes the cognitive resources of working memory (Pisoni, 2000) and selective attention (Shinn-Cunningham and Best, 2008). This would also imply to interpret the observed alpha modulations as relative increases in alpha power for more degraded stimuli. Foxe et al. (1998) report a 10-Hz parieto-occipital enhancement that was stronger for selective attention to auditory cues, which is in line with a role of enhanced alpha oscillations in inhibiting task-irrelevant information - a case we observe for more degraded speech stimuli.

A direct hypothesis that follows from the assumption that degraded speech is taxing cognitive resources would be the following:

\section{Challenges to the auditory system arising from signal degradation trigger increased alpha power}

In the most direct test of this hypothesis, one would use an auditory version of the Sternberg task, where auditory items (e.g., digits) have to be retained in memory for a short retention period before being probed with another auditory digit. Parametrically varying the set size of items to be retained in memory should parametrically increase alpha power during retention (cf. Jensen et al., 2002; Leiberg et al., 2006). However, additional parametric degradation of the speech signal quality in which the digits are presented, should have a similar effect on the retention-phase alpha increase, if degraded speech in itself is taxing the working memory system. Figure 8 illustrates findings from a first experiment we ran on the parametric combination of signal degradation and memory load, using MEG. The alpha power during retention exhibits two clear main effects of both manipulations: More items to retain in memory and a worse signal quality (i.e., less spectral fidelity due to less vocoding bands) both elicit monotonic increases in alpha power (Obleser et al., in preparation).
Both experiments briefly presented here deliver initial evidence that, first, alpha oscillations and changes in alpha power are susceptible to speech signal quality, with relative power increases for more severely degraded speech. This is seen not only during retention, but also in early stimulus encoding (discernible in the 100-200 ms time range in the word comprehension experiment, Figure 7, and also observed during the encoding or digit presentation phase in our auditory Sternberg task, not shown).

Second, alpha modulations - whether originating from the auditory and perisylvian cortex (single subject; Figure 7) or primarily from more domain-general systems such as the superior parietal cortex (group data; Obleser and Weisz, in preparation) - can be indicative of speech comprehension success. Own preliminary data also show that the late alpha suppression after hearing word stimuli can be used to correctly classify low from high likelihood of comprehension when using machine learning algorithms (Obleser and Weisz, in preparation). Also, alpha oscillations tend to have very good signal/noise ratios, allowing single-subject and even singletrial assessment. All of this renders auditorily modulated alpha a particularly useful parameter for populations such as children with hearing deficits or cochlear implant patients, where inference in the single participant is critical.

\section{CONCLUSION AND FUTURE DIRECTIONS}

The intention of the present paper is to promote the research area of "auditory alpha" as an interesting domain in its own right. For this purpose it has to be firstly established that such alpha-like rhythms indeed exist in auditory brain regions that are not an epiphenomenon of the superficially more dominant alpha rhythms in visual and sensori- and motor areas. Even though first evidence has been brought up almost 15 years ago, some skepticism still exists with regard to this issue. We assume that this is partly due to the heavy under investigation of the auditory system as compared to the visual modality. In fact intracranial measurements unequivocally

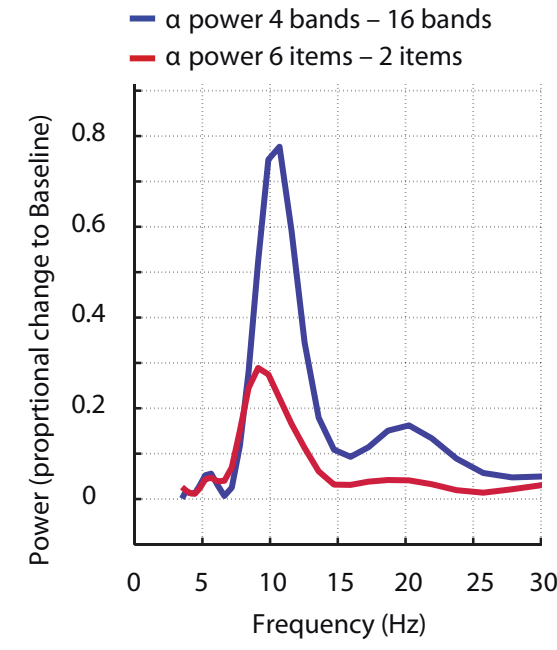

FIGURE 8|Alpha power enhancement (MEG, $n=15$ ) during memory retention in an auditory task is not specific to memory load but is also driven by signal degradation. More severe speech degradation and an increase in

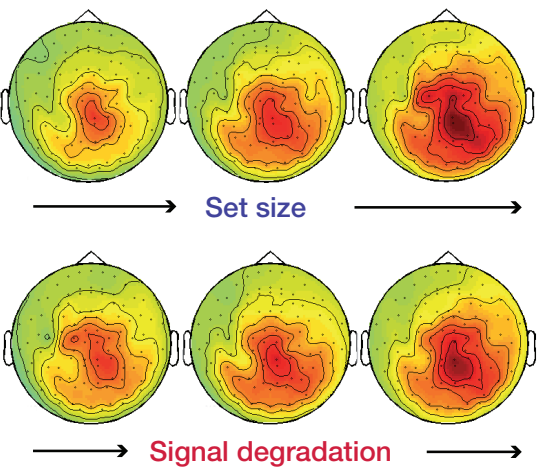

memory set size have comparable effects on the increase in alpha power. NB the difference in alpha peak frequency did not attain significance. The parametric change in beta power $(20 \mathrm{~Hz})$ is only significant in the set size manipulation. 
point to the existence of "auditory alpha" and in this paper we provided additional evidence to the aforementioned pioneering study (Lehtelä et al., 1997), that this activity can be uncovered using non-invasive neuroelectrical methods. An interesting aspect that will need further research in future is the fact that depending on the task and/or method, the "alpha" rhythm can become manifest in slightly varying frequency ranges (from 6 to $12 \mathrm{~Hz}$ ). At this point, based on the scarce literature on auditory alpha it would be premature to ascribe a functional meaning to this observation, but should be revisited to once the empirical basis grows.

With regards to the functional role of alpha, our data so far indicate that it plays a similar role in the auditory domain as in the other sensory and motor modalities. Experimental modulations via selective attention, working memory, stimulus salience alter the level of alpha activity in auditory cortex, and/or speech relevant regions in a manner consistent with observations made in other modalities. With more data from the auditory domain it may be therefore possible to speak of alpha as a rhythm that "universally" (i.e., across modalities) reflects the current state of the E-I balance

\section{REFERENCES}

Andersson, G., Juris, L., Classon, E., Fredrikson, M., and Furmark, T. (2006). Consequences of suppressing thoughts about tinnitus and the effects of cognitive distraction on brain activity in tinnitus patients. Audiol. Neurootol. 11, 301-309.

Andrews, T. J., Halpern, S. D., and Purves, D. (1997). Correlated size variations in human visual cortex, lateral geniculate nucleus, and optic tract. J. Neurosci. 17, 2859-2868.

Artacho-Perula, E., Arbizu, J., ArroyoJimenez Mdel, M., Marcos, P., Martinez-Marcos, A., Blaizot, X., and Insausti, R. (2004). Quantitative estimation of the primary auditory cortex in human brains. Brain Res. $1008,20-28$.

Bastiaansen, M., and Hagoort, P. (2006). Oscillatory neuronal dynamics during language comprehension. Prog. Brain Res. 159, 179-196.

Buzsaki, G., Kaila, K., and Raichle, M. (2007). Inhibition and brain work. Neuron 56, 771-783.

Capotosto, P., Babiloni, C., Romani, G. L., and Corbetta, M. (2009). Frontoparietal cortex controls spatial attention through modulation of anticipatory alpha rhythms. $J$. Neurosci. 29, 5863-5872.

Cherry, E. C. (1953). Some experiments on the recognition of speech, with one and with two ears. J. Acoust. Soc. Am. 25, 975-979.

Crick, F., and Koch, C. (2003). A framework for consciousness. Nat. Neurosci. 6, 119-126.

Dehaene, S., Changeux, J. P., Naccache, L., Sackur, J., and Sergent, C. (2006). Conscious, preconscious, and subliminal processing: a testable taxonomy. Trends Cogn. Sci. 10, 204-211. De Ridder, D., De Mulder, G., Verstraeten, E., Seidman, M., Elisevich, K., Sunaert, S., Kovacs, S., Van der Kelen, K., Van de Heyning, P., and Moller, A. (2007). Auditory cortex stimulation for tinnitus. Acta Neurochir. Suppl.97(Pt2),451-462.

Dohrmann, K., Elbert, T., Schlee, W., and Weisz, N. (2007). Tuning the tinnitus percept by modification of synchronous brain activity. Restor. Neurol. Neurosci. 25, 371-378.

Eggermont, J. J., and Roberts, L. E. (2004). The neuroscience of tinnitus. Trends Neurosci. 27, 676-682.

Elgoyhen, A. B., and Langguth, B. (2010). Pharmacological approaches to the treatment of tinnitus. Drug Discov. Today 15, 300-305.

Foxe, J. J., Simpson, G. V., and Ahlfors, S. P. (1998). Parieto-occipital approximately $10 \mathrm{~Hz}$ activity reflects anticipatory state of visual attention mechanisms. Neuroreport 9 , 3929-3933.

Fu, K. M., Foxe, J. J., Murray, M. M., Higgins, B. A., Javitt, D. C., and Schroeder, C. E. (2001). Attentiondependent suppression of distracter visual input can be cross-modally cued as indexed by anticipatory parieto-occipital alpha-band oscillations. Brain Res. Cogn. Brain Res. 12, 145-152.

Goebel, G., and Hiller, W. (1994). The tinnitus questionnaire. A standard instrument for grading the degree of tinnitus. Results of a multicenter study with the tinnitus questionnaire. HNO 42, 166-172.

Gross, J., Schnitzler, A., Timmermann, L., and Ploner, M. (2007). Gamma

in the underlying neuronal tissue, a claim that currently largely rests on inferring from the visual modality. However, we also showed that research on the auditory modality can add new ideas to the notion of ongoing alpha activity. Based on our work on tinnitus, we forward the idea that already spontaneous alpha activity actually reflects an ongoing inhibitory mechanism, necessary to maintain a functional E-I level and thereby suppressing "spontaneous synchronization." It is noteworthy that a visual equivalent to tinnitus does not seem to exist, however phantom sensation in the somatosensory modality are frequently reported. It will be interesting in future to compare alterations in the pattern of spontaneous activity across modalities in order derive putative common principles with regards to the role of oscillatory activity.

\section{ACKNOWLEDGMENTS}

The works contributing to this review have been supported by the Deutsche Forschungsgemeinschaft (Nathan Weisz), the Max Planck Gesellschaft (Jonas Obleser), and the Tinnitus Research Initiative (Nathan Weisz).

oscillations in human primary somatosensory cortex reflect pain perception. PLoS Biol. 5, e133. doi: 10.1371/journal.pbio.0050133

Gusnard, D. A., Raichle, M. E., and Raichle, M. E. (2001). Searching for a baseline: functional imaging and the resting human brain. Nat. Rev. Neurosci. 2, 685-694.

Haegens, S., Osipova, D., Oostenveld, R., and Jensen, O. (2010). Somatosensory working memory performance in humans depends on both engagement and disengagement of regions in a distributed network. Hum. Brain Mapp. 31, 26-35.

Hallett, M. (2007). Transcarnial magnetic stimulation: a primer. Neuron 55, 187-199.

Hartmann, T., Schulz, H., and Weisz, N. (2011). Probing brain states in realtime: introducing the ConSole environment. Front. Psychology 2:36. doi: 10.3389/fpsyg.2011.00036.

Hauptmann, C., and Tass, P. A. (2010). Restoration of segregated, physiological neuronal connectivity by desynchronizing stimulation. J. Neural Eng. 7, 056008.

Jeanmonod, D., Magnin, M., and Morel, A. (1996). Low-threshold calcium spike bursts in the human thalamus. Common physiopathology for sensory, motor and limbic positive symptoms. Brain 119(Pt 2), 363-375.

Jensen, O., Gelfand, J., Kounios, J., and Lisman, J. E. (2002). Oscillations in the alpha band $(9-12 \mathrm{~Hz})$ increase with memory load during retention in a short-term memory task. Cereb. Cortex 12, 877-882.

Jensen, O., Kaiser, J., and Lachaux, J. P. (2007). Human gamma-frequency oscillations associated with attention and memory. Trends Neurosci. 30, 317-324.

Jensen, O., and Mazaheri, A. (2010). Shaping functional architecture by oscillatory alpha activity: gating by inhibition. Front. Hum. Neurosci. 4:186. doi: 10.3389/ fnhum.2010.00186

Jokisch, D., and Jensen, O. (2007). Modulation of gamma and alpha activity during a working memory task engaging the dorsal or ventral stream. J. Neurosci. 27, 3244-3251.

Kerlin, J. R., Shahin, A. J., and Miller, L. M. (2010). Attentional gain control of ongoing cortical speech representations in a "cocktail party". J. Neurosci. 30, 620-628.

Kleinjung, T., Vielsmeier, V., Landgrebe, M., Hajak, G., and Langguth, B. (2008). Transcranial magnetic stimulation: a new diagnostic and therapeutic tool for tinnitus patients. Int. Tinnitus J. 14, 112-118.

Klimesch, W., Sauseng, P., and Hanslmayr, S. (2007). EEG alpha oscillations: the inhibition-timing hypothesis. Brain Res. Rev. 53, 63-88.

Lamme, V.A. (2006). Towards a true neural stance on consciousness. Trends Cogn. Sci. 10, 494-501.

Lehtelä, L., Salmelin, R., and Hari, R. (1997). Evidence for reactive magnetic $10-\mathrm{Hz}$ rhythm in the human auditory cortex. Neurosci. Lett. 222, 111-114.

Leiberg, S., Lutzenberger, W., and Kaiser, J. (2006). Effects of memory load on cortical oscillatory activity during auditory pattern working memory. Brain Res. 1120, 131-140.

Llinas, R. R., Ribary, U., Jeanmonod, D., Kronberg, E., and Mitra, P. P. 
(1999). Thalamocortical dysrhythmia: a neurological and neuropsychiatric syndrome characterized by magnetoencephalography. Proc. Natl. Acad. Sci. U.S.A. 96, 15222-15227.

Lorenz, I., Muller, N., Schlee, W., Hartmann, T., and Weisz, N. (2009). Loss of alpha power is related to increased gamma synchronization - a marker of reduced inhibition in tinnitus? Neurosci. Lett. 453, 225-228.

Lorenz, I., Muller, N., Schlee, W., Langguth, B., and Weisz, N. (2010). Short-term effects of single repetitive TMS sessions on auditory evoked activity in patients with chronic tinnitus. J. Neurophysiol. 104, 1497-1505.

Meeuwissen, E. B., Takashima, A., Fernández, G., and Jensen, O. (2011). Increase in posterior alpha activity during rehearsal predicts successful long-term memory formation of word sequences. Hum. Brain. Mapp. 32, doi: 10.1002/hbm.21167. [Epub ahead of print].

Moazami-Goudarzi, M., Michels, L., Weisz, N., and Jeanmonod, D. (2010). Temporo-insular enhancement of EEG low and high frequencies in patients with chronic tinnitus. QEEG study of chronic tinnitus patients. BMC Neurosci. 11, 40 . doi: 10.1186/1471-2202-11-40

Norena, A., Micheyl, C., Chery-Croze, S., and Collet, L. (2002). Psychoacoustic characterization of the tinnitus spectrum: implications for the underlying mechanisms of tinnitus. Audiol. Neurootol. 7, 358-369.

Norena, A. J., and Eggermont, J. J. (2003). Changes in spontaneous neural activity immediately after an acoustic trauma: implications for neural correlates of tinnitus. Hear. Res. 183, 137-153.

Obleser, J., Eisner, F., and Kotz, S. A. (2008). Bilateral speech comprehension reflects differential sensitivity to spectral and temporal features. $J$. Neurosci. 28, 8116-8123.

Obleser, J., and Kotz, S.A. (2010). Multiple brain signatures of integration in the comprehension of degraded speech. Neuroimage 55, 713-723.

Obleser, J., and Weisz, N. (2010). "Differential influences of spectral and temporal features of speech on human oscillatory brain dynamics," in Paper Presented at the Neuroscience 2010, San Diego.
Okamoto, H., Stracke, H., Stoll, W., and Pantev, C. (2010). Listening to tailormade notched music reduces tinnitus loudness and tinnitus-related auditory cortex activity. Proc. Natl. Acad. Sci. U.S.A. 107, 1207-1210.

Ortmann, M., Muller, N., Schlee, W., and Weisz, N. (2011). Rapid increases of gamma power in the auditory cortex following noise trauma in humans. Eur. J. Neurosci. 33, 568-575.

Osipova, D., Hermes, D., and Jensen, O. (2008). Gamma power is phaselocked to posterior alpha activity. PLoS ONE 3, e3990. doi: 10.1371/journal. pone.0003990

Perruchet, P. (1985). A pitfall for the expectancy theory of human eyelid conditioning. Pavlov. J. Biol. Sci. 20 , 163-170.

Pisoni, D. B. (2000). Cognitive factors and cochlear implants: some thoughts on perception, learning, and memory in speech perception. Ear Hear. 21, 70-78.

Posner, M. I., and Petersen, S. E. (1990). The attention system of the human brain. Annu. Rev. Neurosci. 13, 25-42.

Rihs, T. A., Michel, C. M., and Thut, G. (2007). Mechanisms of selective inhibition in visual spatial attention are indexed by alpha-band EEG synchronization. Eur. J. Neurosci. 25, 603-610.

Schlee, W., Hartmann, T., Langguth, B., and Weisz, N. (2009a). Abnormal resting-state cortical coupling in chronic tinnitus. BMC Neurosci. 10, 11. doi: 10.1186/1471-2202-10-11

Schlee, W., Mueller, N., Hartmann, T., Keil, J., Lorenz, I., and Weisz, N. (2009b). Mapping cortical hubs in tinnitus. BMC Biol. 7, 80. doi: 10.1186/1741-7007-7-80

Schroeder, C. E., and Lakatos, P. (2009). The gamma oscillation: master or slave? Brain Topogr. 22, 24-26.

Scott, S. K., Blank, C. C., Rosen, S., and Wise, R. J. (2000). Identification of a pathway for intelligible speech in the left temporal lobe. Brain 123(Pt 12), 2400-2406.

Shahin, A. J., Picton, T. W., and Miller, L. M. (2009). Brain oscillations during semantic evaluation of speech. Brain Cogn. 70, 259-266.

Shannon, R. V., Zeng, F. G., Kamath, V., Wygonski, J., and Ekelid, M. (1995). Speech recognition with primarily temporal cues. Science 270, 303-304.
Shinn-Cunningham, B. G., and Best, V. (2008). Selective attention in normal and impaired hearing. Trends Amplif. 12, 283-299.

Smith, D. T., Jackson, S. R., and Rorden, C. (2009). Repetitive transcranial magnetic stimulation over frontal eye fields disrupts visually cued auditory attention. Brain Stimul. 2, 81-87.

Spierer, L., Bellmann-Thiran, A., Maeder, P., Murray, M. M., and Clarke, S. (2009). Hemispheric competence for auditory spatial representation. Brain 132, 1953-1966.

Sterman, M. B., and Friar, L. (1972). Suppression of seizures in an epileptic following sensorimotor EEG feedback training. Electroencephalogr. Clin. Neurophysiol. 33, 89-95.

Thut, G., Nietzel, A., Brandt, S. A., and Pascual-Leone,A. (2006). Alpha-band electroencephalographic activity over occipital cortex indexes visuospatial attention bias and predicts visual target detection. J. Neurosci. 26 , 9494-9502.

Tiihonen, J., Hari, R., Kajola, M., Karhu, J., Ahlfors, S., and Tissari, S. (1991). Magnetoencephalographic $10-\mathrm{Hz}$ rhythm from the human auditory cortex. Neurosci. Lett. 129, 303-305.

van der Loo, E., Gais, S., Congedo, M., Vanneste, S., Plazier, M., Menovsky, T., Van de Heyning, P., and De Ridder, D. (2009). Tinnitus intensity dependent gamma oscillations of the contralateral auditory cortex. PLoS ONE 4, e7396. doi: 10.1371/journal. pone.0007396

van Dijk, H., Nieuwenhuis, I. L., and Jensen, O. (2010). Left temporal alpha band activity increases during working memory retention of pitches. Eur. J. Neurosci. 31, 1701-1707.

van Dijk, H., Schoffelen, J. M., Oostenveld, R., and Jensen, O. (2008). Prestimulus oscillatory activity in the alpha band predicts visual discrimination ability. J. Neurosci. 28, 1816-1823.

van Dijk, J. G., Thijs, R. D., Benditt, D. G., and Wieling, W. (2009). A guide to disorders causing transient loss of consciousness: focus on syncope. Nat. Rev. Neurol. 5, 438-448.

Weisz, N., Dohrmann, K., and Elbert, T. (2007a). The relevance of spontaneous activity for the coding of the tinnitus sensation. Prog. Brain Res. 166, 61-70.

Weisz, N., Muller, S., Schlee, W., Dohrmann, K., Hartmann, T., and Elbert, T. (2007b). The neural code of auditory phantom perception. J. Neurosci. 27, 1479-1484.

Weisz, N., Lecaignard, F., Müller, N., and Bertrand, B. (2011). The modulatory influence of a predictive cue on the auditory steady-state response. Hum. Brain Mapp. (in press).

Weisz, N., Moratti, S., Meinzer, M., Dohrmann, K., and Elbert, T. (2005). Tinnitus perception and distress is related to abnormal spontaneous brain activity as measured by magnetoencephalography. PLoS Med. 2, e153. doi: 10.1371/journal. pmed.0020153

Worden, M. S., Foxe, J. J., Wang, N., and Simpson, G. V. (2000). Anticipatory biasing of visuospatial attention indexed by retinotopically specific alpha-band electroencephalography increases over occipital cortex. $J$. Neurosci. 20, RC63.

Zatorre, R. J., Belin, P., and Penhune, V. B. (2002). Structure and function of the auditory cortex: music and speech. Trends Cogn. Sci. 6, 37-46.

Zatorre, R. J., and Penhune, V. B. (2001). Spatial localization after excision of human auditory cortex. J. Neurosci. 21, 6321-6328.

Zeki, S., and Bartels, A. (1999). Toward a theory of visual consciousness. Conscious. Cogn. 8, 225-259.

Conflict of Interest Statement: The authors declare that the research was conducted in the absence of any commercial or financial relationships that could be construed as a potential conflict of interest.

Received: 14 January 2011; paper pending published: 03 February 2011; accepted: 05 April 2011; published online: 26 April 2011. Citation: Weisz N, Hartmann T, Müller $N$, Lorenz I and Obleser J (2011) Alpha rhythms in audition: cognitive and clinical perspectives. Front. Psychology 2:73. doi: 10.3389/fpsyg.2011.00073

This article was submitted to Frontiers in Perception Science, a specialty of Frontiers in Psychology.

Copyright (C) 2011 Weisz, Hartmann, Müller, Lorenz and Obleser. This is an openaccess article subject to a non-exclusive license between the authors and Frontiers Media SA, which permits use, distribution and reproduction in other forums, provided the original authors and source are credited and other Frontiers conditions are complied with. 